\title{
Editorial: The Future of Physical Review D
}

I would like to introduce myself as the recently appointed Lead Editor of Physical Review D. I am honored to be appointed to this position, which was served so well by Erick Weinberg for the past 23 years. I would like to thank him for his tremendous service to the global community of readers and authors of the journal. These are very big shoes to fill, indeed.

I am looking forward to working with the dedicated Editors and the Editorial Board of Physical Review D to advance the journal's key role as a leading journal of the American Physical Society (APS). I am extremely proud of their dedication to the rigorous editorial process which strives for high publishing standards, rigor, fairness, and collegiality among authors, referees, and Editors.

The journal serves the broad scientific community, ranging from theoretical high energy physics, including formal theory, to broad fields of experimental high energy physics, gravitation, cosmology, and astroparticle physics. Importantly, papers in the broad field of high energy physics are published in partnership with $\mathrm{SCOAP}^{3}$ and are therefore published under open access without any direct charges to the authors. This open-access model provides high visibility and a wide readership for published articles in the field. It also showcases APS's continuing leadership in open-access publishing.

My goal is to build and expand on these strengths which I believe represent fundamental values of scientific publishing for our community. In order to uphold and improve the status of Physical Review $D$ as a top-tier journal in its respective fields, we are reaffirming its high publishing standards by adhering to the criteria of importance, high quality, and innovativeness of published papers. In cases, when quality assessments can be made at the initial stages of the reviewing process, some editorial decisions, in consultation with the Editorial Board, can be made directly.

While our team continues to support publishing in well-represented core areas of the journal, we also plan to expand our outreach in directions at the interface of high energy physics and forefront developments in other areas of physics. We aim to further increase the journal's impact by proactively seeking to publish significant and important results. To achieve these goals, we will increase our efforts to reach out to different research communities through increased communications with institutions and active participation in both general and topical conferences by the Editors and the members of the Editorial Board.

And above all, I would like to reach out to you, our community of readers, authors, and referees, and urge you to publish your very best research in Physical Review $D$. This a key and a crucial step toward the goals we strive for at Physical Review $D$ to be a nonprofit, leading journal of, by, and for our community.

\section{Mirjam Cvetič}

Lead Editor, Physical Review D

American Physical Society

Published 13 July 2020

DOI: 10.1103/PhysRevD.102.010001 